\title{
Turbulent bubble jets in microgravity. Spatial dispersion and velocity fluctuations
}

\author{
Pau Bitlloch · Xavier Ruiz · Laureano Ramírez-Piscina · Jaume Casademunt
}

Received: date / Accepted: date

\begin{abstract}
A detailed statistical analysis of bubble disper2 sion in turbulent jets based on data from drop tower exper3 iments is presented here. A stochastic model is also intro4 duced in order to capture these statistics to a large extent, 5 treating bubbles as passive tracers with a local diffusivity given by a $k-\varepsilon$ description of the turbulence. Bubble-bubble and bubble-flow interactions are neglected. Simple scaling analysis suggests that this approach is justified sufficiently 9 far downstream. It is also found that, although interactions cannot be neglected very close to the inlet, the model pre1 dictions for the overall spatial distribution of the bubble ensemble are compatible with data within experimental uncer3 tainty, and within the limited statistics of the experiments. In addition, the velocity fluctuations from the same experiments is analyzed, obtaining the local standard deviation of bubble velocities. We also find good agreement between experimental data and the effective model. Slight deviations between the model predictions and the experimental data
\end{abstract}

P. Bitlloch ( ) J J. Casademunt

Departament d'Estructura i Constituent de la Matèria,

Universitat de Barcelona,

Av. Diagonal 647, 08028-Barcelona, Spain

E-mail: pau.bitlloch@gmail.com

\section{Ruiz}

Departament de Química, Física i Inorgànica,

Universitat Rovira i Virgili,

Marcelí Domingo s/n, 43007-Tarragona, Spain

E-mail: josepxavier.ruiz@urv.net

L. Ramírez-Piscina

Departament de Físca Aplicada,

Universitat Politècnica de Catalunya,

Doctor Marañón 44, 08028-Barcelona, Spain

E-mail: laure@fa.upc.edu

X. Ruiz · L. Ramírez-Piscina · J. Casademunt ( ) Institut d'Estudis Espacials de Catalunya,

Gran Capità 2-4, 08034-Barcelona, Spain

E-mail: jaume.casademunt@ub.edu
19 are found at the jet margins, concerning the dependence on 20 Reynolds number of jet angle and the relative velocity fluc21 tuations. Consequently, significant bubble-flow interactions 22 seem to be confined at the boundaries of the jets.

23 Keywords turbulent jet · bubble dispersion - bubble inter24 actions $\cdot$ microgravity $\cdot$ drop tower $\cdot$ velocity fluctuations

\section{Introduction}

26 The management and control of two phase flows in microgravity is a key area of research in space technology, due 28 to direct applicability in critical areas such as life support 29 systems, power generation and propulsion, or thermal manso agement with gas-liquid heat exchangers (National Research ${ }_{31}$ Council of the National Academies, 2012). In the study of 32 dynamics of bubbly flows, a common problem is the dif3 ficulty of controlling the characteristics and the regularity 34 of the generation of bubbles when no buoyancy is present. 35 In this respect, Carrera et al (2008) introduced the strategy 36 of injecting previously-formed liquid-gas slug flows into a 37 liquid cavity, instead of injecting gas directly, with the idea 38 that creating the bubbles prior to injection would allow a bet39 ter control of the bubble formation mechanism in a gravity40 insensitive manner. Specifically, the method consisted of in41 jecting gas into a liquid cross-flow in capillary T-junction, 42 to form a regular slug flow, and then inject the slug flow 43 into a cavity. Indeed, the methods produced very periodic 44 trains of bubbles of a prescribed size, as opposed to direct 45 injection of gas into a liquid cavity, which in the absence 46 of gravity produces in general much more dispersed bubble 47 sizes. Carrera et al (2008) established the theoretical basis 48 for the mechanism by which bubbles were detached by the 49 drag forces of the liquid crossflow competing with the capil50 lary forces, being buoyancy forces negligible in comparison 51 to the other two. The outcome was then independent of the 


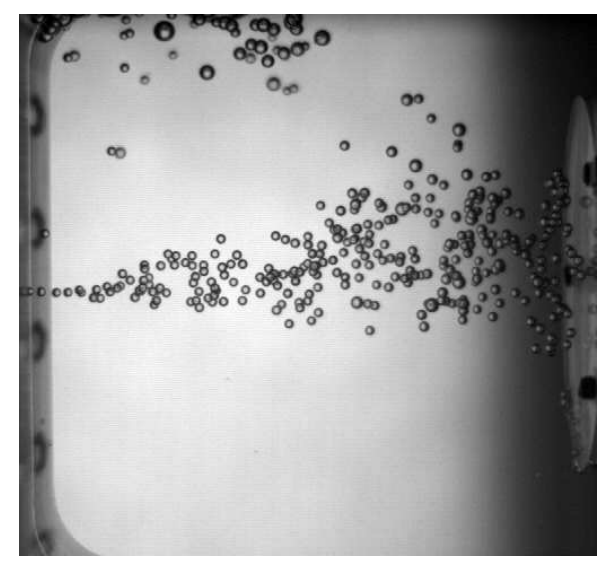

Fig. 1 Snapshot of a typical experiment of slug bubble injection after 2.5 seconds of microgravity.

52 level of gravity. The size of the bubbles generated with this 53 procedure was typically of the order of the diameter of the 54 capillary tube, but it could be slightly tuned. Altogether with

55 the bubble formation frequency and the bubble-bubble dis5 tance, their size could also be modified by adjusting the liquid and gas injection rates into the T-junction. Detailed characterization of the performance of this two-phase T-junction was exhaustively studied by Arias et al (2009, 2010).

Carrera et al (2008) also conducted a series of micro-

61 gravity experiments in the drop tower of ZARM in Bremen, 111 The experimental setup was already described by Carrera in which this bubble generator was used for the first time in ${ }_{112}$ et al (2008). It consists of a cavity of $100 \times 100 \times 100 \mathrm{~mm}^{3}$, microgravity. They created and injected uniform slug flows 113 as sketched in Fig. 2, in which a bubble jet (air in water) is 64 into a quiescent cubic cavity. In the absence of buoyancy ef- ${ }_{114}$ generated through the injection of a slug flow. This slug flow fects, the injection of the slug flow resulted in the formation ${ }_{115}$ is previously formed in a $1.5 \mathrm{~mm}$ diameter T-junction. The of a turbulent jet across the cavity, in which bubbles were ${ }_{116}$ bubble diameter depends on the injection parameters but it ${ }_{67}$ dispersed in a roughly conical shape (Fig. 1). While the ve- 117 is roughly of the order of the capillary. In Fig. 2 we also ${ }_{68}$ locity field of the carrying fluid could not be visualized, the 118 show the structure of a typical mean velocity field obtained jet region occupied by the bubbles appeared statistically sta- ${ }_{119}$ numerically in the case of a single-phase flow.

tionary once formed, although the axial symmetry was lost 120

The analysis of the experimental results will focus on due to the remnants of the flow generated by the rising bub- 121 the position and instantaneous velocity of all bubbles for 72 bles at the 1g stage prior to microgravity. Remarkably, with 122 the most typical and well behaved experiments, correspond${ }_{3}$ the use of this injection method, the size distribution of the ${ }_{123}$ ing to the cases with $R e=690$ (with parameters of injection ${ }_{74}$ injected bubbles was highly monodisperse and easily con- ${ }_{124} Q_{l}=41 \mathrm{ml} / \mathrm{min}$, and $Q_{g}=16 \mathrm{ml} / \mathrm{min}$ and resulting bubbles of trollable even in microgravity. In their paper, Carrera et al ${ }_{125}$ diameter $d_{B} \simeq 1.8 \mathrm{~mm}$ ) and $R e=1170$ (with $Q_{l}=74 \mathrm{ml} / \mathrm{min}$, (2008) showed how the experimental mean velocity of bub- ${ }_{126} \quad Q_{g}=18 \mathrm{ml} / \mathrm{min}$ and $d_{B} \simeq 1.4 \mathrm{~mm}$ ). The definition of Reynolds bles (measured at different points along the axis and at the ${ }_{127}$ number used will be described later in Sec.2.4. More deboundaries of the experimental bubble cones) followed to a 128 tails on the experimental setup can be found in Carrera et al large extent the analytical solution for the averaged turbulent ${ }_{129}$ (2008).

so flow of a liquid jet without any dispersed phase, as described ${ }_{130} \quad$ In order to measure the position and velocity of each 81 by Schlichting (1979). This result implied that the presence 131 bubble during the experiments, all the images taken by the of bubbles did not affect significantly the mean liquid flow, ${ }_{132}$ high speed video camera were processed so that an autos except for an increase of the total injected momentum. Here ${ }_{133}$ matic particle tracking software enabled us to identify the 84 we extend and complete the analysis of the same series of ${ }_{134}$ paths described by all bubbles. To do so, first it was necmicrogravity experiments to the statistics of velocity fluctu- ${ }_{135}$ essary to homogenize the background of all the frames by ations to elucidate to what extent and under what conditions 136 subtracting, to each of them, a picture taken by the same 87 the potential two-way interactions between bubbles and tur- 137 camera in the absence of bubbles. After the background cor${ }_{88}$ bulence can be quantified and/or possibly neglected. To do 138 rection, we used a standard filter to highlight the interphase

so we will introduce an effective stochastic model that ne1 mental data. This model includes the finite-size effects of the ainer and treats the averaged effects of turbulence within with a finite volume method in a 2D axisymmetric mesh. voted to the description of the experimental setup and the stochastic model for an ensemble of passive bubbles that fixes the reference to detect possible deviations from this passive behavior in the turbulent jet. In the next two sections, Secs. 3 and 4 we analyze experimental data regarding the spatial structure of the bubble jet and velocity statistics, and
compare them to the numerical simulations based on the efз fective model. While most of the results are consistent with mental uncertainty, some discrepancies seem to point out to we present a brief discussion and the main conclusions in Sec. 5.

\section{Experimental setup and theoretical model}

\subsection{Experimental setup}




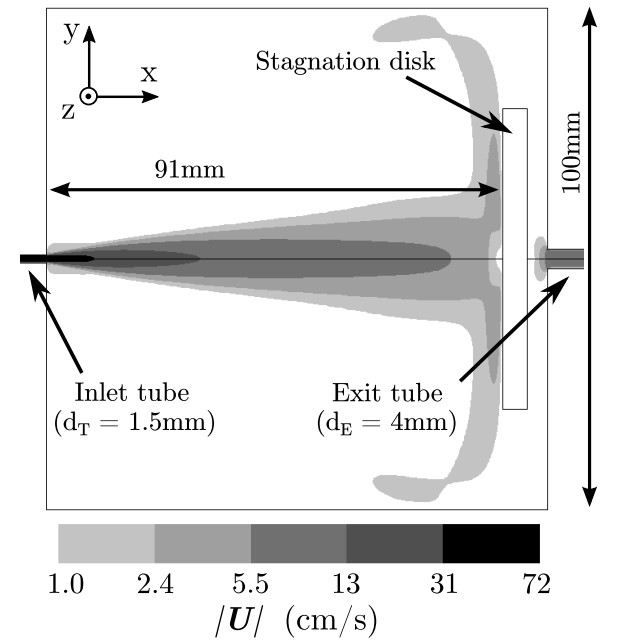

Fig. 2 Experimental cell schematics with contour levels of mean velocity $(\mathrm{cm} / \mathrm{s})$, as obtained by CFD calculation, for a liquid jet with $R e=690$. The origin of the coordinated system is located at the entrance of the cavity in the central axis of the jet.

139 of each bubble. Finally we used particle tracking methods to ${ }_{140}$ identify and follow the trajectories of all bubbles (Bitlloch, 141 2012).

142 Since the experimental data is taken from 2D snapshots ${ }_{143}$ where the real 3D configuration has been projected, some 144 of the information is lost in the process. In the first place, 145 the component of the velocity of bubbles in the direction $z$, 146 perpendicular to the plane of the snapshot, cannot be mea147 sured. This is not a major issue since the main component of ${ }_{17}$ ${ }_{148}$ the velocity is $u_{x}$, in the axial direction $x$. In addition to this, 149 the properties of the flow in the directions $y$ and $z$ should be 150 statistically equivalent. A more serious limitation is the fact 151 that we cannot measure the depth $z$ at which any bubble is 180 152 placed, therefore when we conduct a statistical analysis of 153 bubble velocities, we are inevitably mixing velocities that 154 were in fact at different layers of the jet. This fact will be 155 properly incorporated in the statistical analysis.

1562.2 Description of the single-phase jet

157 In theoretical framework of the so-called $k-\varepsilon$ models, it ${ }_{182}$ and the other constants, i.e.

${ }_{158}$ is assumed that one can decompose the total velocity field

159 of a turbulent flow in two parts, a mean flow component

160 and a fluctuating part. Regarding the first component, it is

161 well known that the spatial structure of the mean flow ve- ${ }^{183}$

162 locity field of a turbulent single-fluid jet is independent of

${ }_{163} \operatorname{Re}$ (Schlichting, 1979). In these models the fluctuating part

164 of the flow is described in terms of two continuous fields, the

${ }_{165}$ local turbulent kinetic energy $k$ of the fluctuating part, and ${ }^{184}$

${ }_{166}$ its dissipation rate $\varepsilon$. Closed transport equations for these

167 fields are then postulated. In the case of interest here for the

$A_{0}=4.04, \quad A_{s}=\sqrt{6} \cos \phi$,

$\phi=\frac{1}{3} \cos ^{-1}\left(\sqrt{6} \frac{E_{i j} E_{j k} E_{k i}}{\left(\sqrt{E_{i j} E_{i j}}\right)^{3}}\right)$,

$C_{2}=1.9, \sigma_{k}=1.0, \sigma_{\varepsilon}=1.2$,

168 application of this approach, namely the study of turbulent 185 have been adjusted to the values that offer an optimal per169 jets, the standard $k-\varepsilon$ model it is known to over predict the 186 formance of the model. 
1872.3 Stochastic model for the bubble jet

188 Since the experimental results of Carrera et al (2008) indi189 cated that the local averaged velocities of bubbles coincide 190 to a good extent to the mean flow velocity field of a turbu191 lent single-fluid from the solution of (Schlichting, 1979), the 92 spreading of the spatial distribution of bubbles must be di- ${ }^{241}$ 3 rectly related to the fluctuating part of the flow. In Fig. 3 we ${ }^{242}$ 94 can appreciate how the streamlines of the flow that are actu- ${ }^{24}$ 5 ally being injected into the cell only suffer a slight opening ${ }^{24}$ ${ }_{96}$ (of no more than twice its initial separation $d_{T}$ ) after the full ${ }^{24}$ 7 length of the jet. It is easy to see how the larger width of ${ }^{246}$ the jet is determined by its external layers, that incorporate ${ }^{247}$ 9 streamlines from the recirculating flow. In addition, turbu- ${ }^{24}$ lence provides a mechanism that mixes all those layers of mean flow, allowing the dispersion of bubbles through them.

Hence, we need to make use of the local characteristics of turbulence in order to properly describe the dispersion of bubbles through the transversal layers of the flow and, at the ${ }^{25}$ same time, to confine them inside the boundaries of the jet, 25 preventing them from freely disperse through the whole ex- ${ }^{25}$ perimental cell.

Within the above-mentioned realizable $k$ - $\varepsilon$ model of turbulence, we will associate a local diffusivity to bubbles that is inherited from the diffusivity of the kinetic energy of the turbulent component of the flow in the absence of bubbles. The main assumption is thus that bubbles are also passive with respect to the fluctuating component of the flow. As mentioned before, this assumption must be correct in principle sufficiently far downstream, where the bubble suspension becomes more and more dilute and the bubble size becomes negligible compared to the scales of the flow.

Since bubbles are not point-like and the number of them 2 219 is relatively small, the aim of the model is to formulate an 262 equation for the probability distribution of finding a bubble 263 221 at a certain location. The model does not intend to be a good 264 description of the individual trajectories of bubbles, which 265 are far from diffusive at small scales of the flow due to strong 266 spatial and temporal correlations of the carrying flow. This 267 sity $P(\mathbf{s}, t)$ of finding a bubble in a certain position at an implies, for instance, that the model will be inappropriate to 268 instant of time. This distribution coincides with the concendescribe properties related to the geometry of the bubble tra- 269 tration of an ensemble of independent bubbles, and is given ${ }_{227}$ jectories themselves or the correlations between them, such 270 by the so-called Fokker-Planck equation associated to the ${ }_{228}$ as the probability of bubble encounters and consequently of 271 stochastic differential equation (11). This equation has the 229 possible coalescence. Despite this limitation of the model, 272 form

230 the assumption of a local diffusivity of the probability of 231 finding bubbles may be reasonably justified to describe the spatial distribution of an ensemble of realizations, provided 33 that coalescence events are rare.

$$
\frac{\partial P(\mathbf{s}, t)}{\partial t}+\nabla \cdot(\mathbf{U} P)=\nabla \cdot\left[D_{p} \nabla P\right]
$$

273 In this framework, the concentration of bubbles, proportional

For the purposes of studying the spatial bubble disper- 274 to the probability distribution $P$, diffuses as a passive scalar 235 sion, the above model pictures the dynamics of bubbles as 275 advected with the mean flow velocity $\mathbf{U}(\mathbf{s}, t)$, but with a dif${ }_{236}$ described by a biased random walk. We write explicitly the ${ }_{276}$ fusion coefficient $D_{p}(\mathbf{s}, t)$ which depends on the local prop${ }_{237}$ instantaneous velocity of a bubble $\mathbf{u}_{B}$ as a stochastic (Lange- 277 erties of the turbulence through the field $k^{2} / \varepsilon$. 


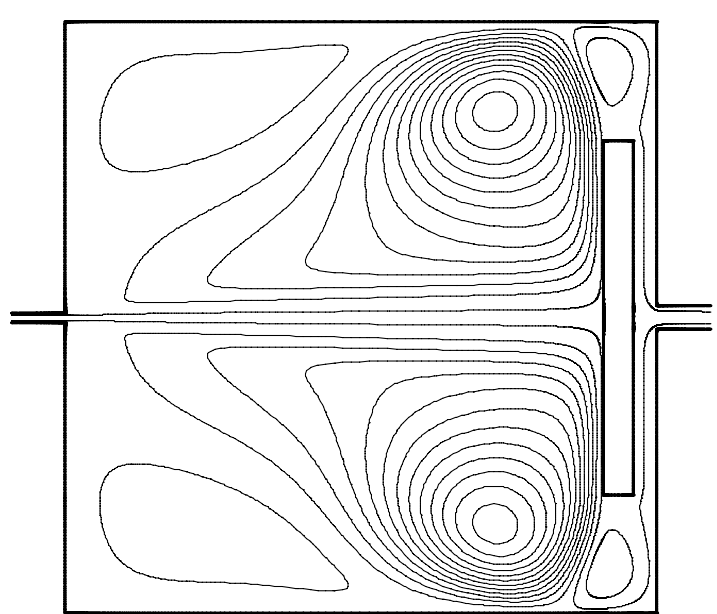

Fig. 3 Streamlines of the mean flow in the experimental cell, obtained from a simulation with axial symmetry.

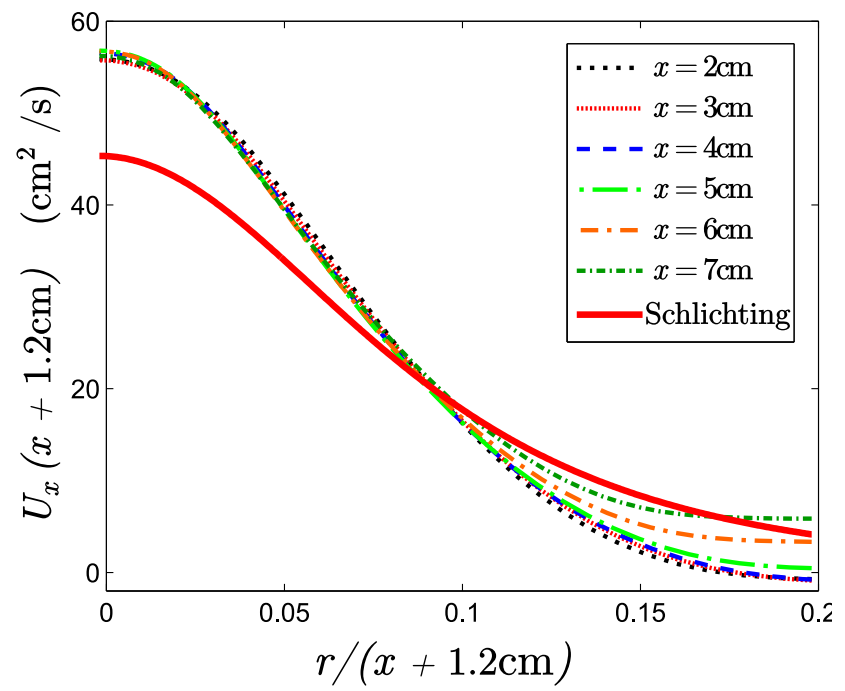

Fig. 4 Collapse of many curves of $U_{x} \cdot\left(x+x_{0}\right)$ for a jet with $R e=$ 690. $r$ is the radial distance in cylindrical coordinates. Dashed lines show the simulation results at various transversal sections of the jet. Solid line corresponds to Schlichting (1979) analytical solution of an infinite single-phase turbulent jet with an infinitely small slit. $x_{0}$ has been adjusted to $1.2 \mathrm{~cm}$ in order to overlap all the curves from the simulations, due to the finite size of the inlet.

2782.4 The mean velocity field

Crien The reported calculations were caried out with the help of 325

279 The reported calculations were carried out with the help of 325

280 the commercial software FLUENT, using a 2D axisymmet-

281 ric mesh consisting of 56100 cells arranged in a non-uniform ${ }_{32}$

282 distribution, in order to obtain higher density of cells in the

283 critical areas with higher gradients of flow velocity. The vol- 329

284 umes for the cells ranged from $3 \cdot 10^{-13} \mathrm{~m}^{3}$ up to $2 \cdot 10^{-7}{ }_{330}$

${ }_{285} \mathrm{~m}^{3}$ (of the total $7.7 \cdot 10^{-4} \mathrm{~m}^{3}$ ). Tests of different meshes

${ }_{286}$ with various cell densities were performed without notice-

287 able variations in the outcome.
Taking into account that the presence of bubbles in the 289 injector increases the velocity of the liquid slugs between 290 them, for the simulation of the effective single-phase jet the 291 effective injected momentum $J$ is defined as

$$
J=\rho_{l} Q_{l}\left\langle U_{T}\right\rangle=Q_{l}\left(Q_{l}+Q_{g}\right) \frac{\rho_{l}}{A_{T}},
$$

${ }_{292} Q_{l}$ and $Q_{g}$ being respectively the volumetric flow rates of 293 liquid and gas injected into the T-junction. Note that the ef294 fect of the presence of bubbles is here reduced to a modifi295 cation of the injected momentum, but the medium is treated 296 as an effective continuum, so the intermittent presence of 297 bubbles at any a given point is lost.

298 The appropriate definition of Reynolds number for the 299 jet will be given by taking the characteristic length $L_{\mathrm{c}}$ as the local diameter of the jet at any position, and the character301 istic velocity at this same position as given by the injected 302 momentum $U_{\mathrm{c}}=\sqrt{J /\left(\rho_{l} A_{\mathrm{c}}\right)}$, with $A_{\mathrm{c}}=\pi L_{\mathrm{c}}^{2} / 4$, which leads 303 to

$$
R e=\frac{L_{\mathrm{c}}}{v} \sqrt{\frac{J}{\rho_{l} A_{\mathrm{c}}}}=\frac{4 \sqrt{Q_{l}\left(Q_{l}+Q_{g}\right)}}{\pi v d_{T}},
$$

304 with $d_{T}$ the diameter of the inlet, which coincides in the ex305 periments with that of the T-junction capillary tubes.

306 It is worth remarking that the effective Reynolds number 307 of the jet, in principle depending on the characteristic scales 8 of length $L_{\mathrm{c}}$ and velocity $U_{\mathrm{c}}$ whose local values vary with 9 the distance from the injection point, remains constant all along the jet. This can be easily shown (Schlichting, 1979) 31 by observing experimentally that the opening angle of a tur2 bulent jet remains constant with the distance, while on the 3 other hand, the flow velocity scale is inversely proportional to the distance. This causes that, to a first approximation, the turbulent flow is statistically self-similar along the jet, under the appropriate rescaling of length and time, i.e. the characteristic eddy velocities are being reduced downstream in the same proportion as their size increases.

In Fig. 2 and 3 we show the structure of the mean velocity field for the single-phase turbulent jet as computed within 321 the $k-\varepsilon$ model. In these figures it is easy to see how the fi322 nite size of the experimental cell plays an important role in 323 the flow structure, specially in the areas with strong recir224 culation and near the stagnation disk. In Fig. 4 we display the results obtained from the numerical integration of the model for the radial variation of the axial velocity at different positions along the jet axis, and compare with the analytical Schlichting solution for an infinite system. We can observe how the numerical solution of the jet presents a sharper opening angle than the case of the solution for an infinite system, with a significantly higher velocity at the jet axis for 32 the former, given the same injected momentum. Despite this 333 finite-size effect on the opening angle, the jet maintains its 


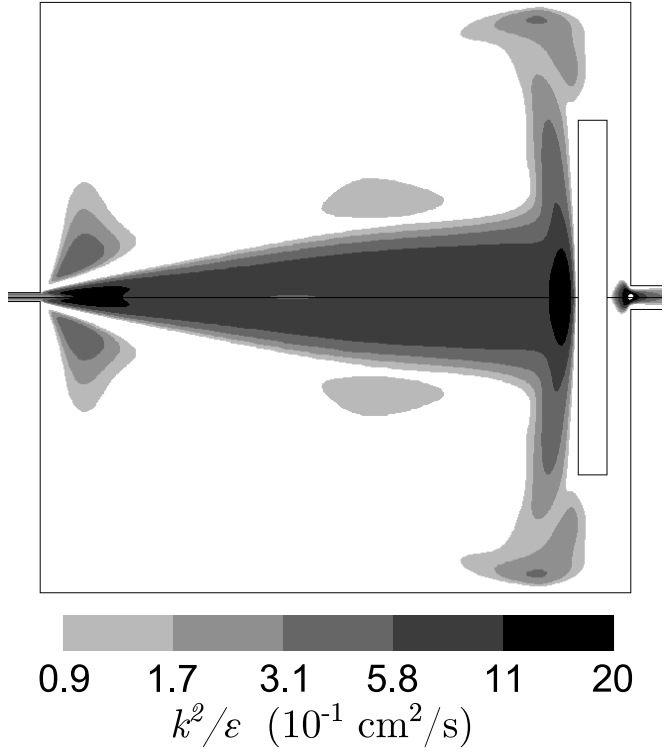

Fig. 5 Contours of constant $k^{2} / \varepsilon \quad\left(10^{-1} \mathrm{~cm}^{2} / \mathrm{s}\right)$, as obtained by CFD calculations.

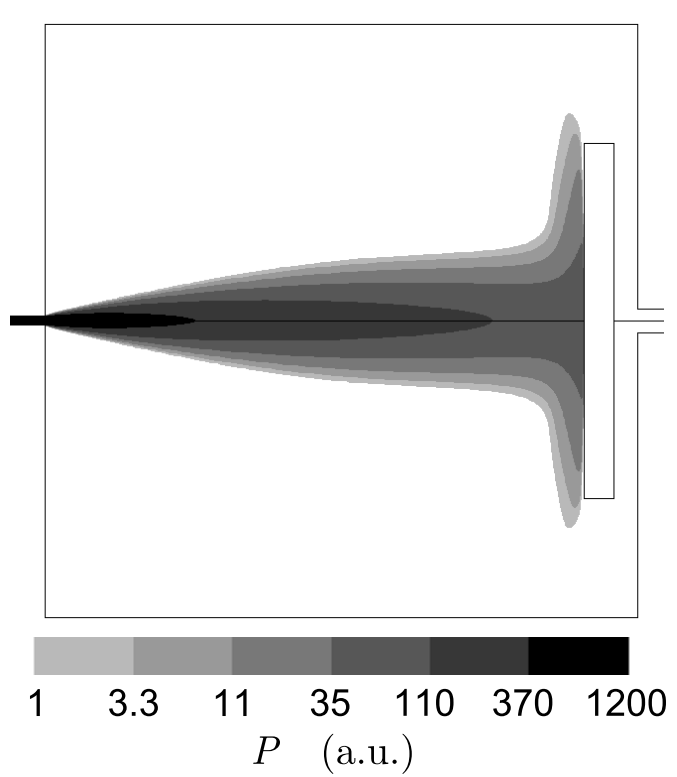

340 2.5 The bubble-turbulence coupling

${ }_{341}$ Since the bubble size is not only monodisperse but preserved 342 through the evolution, while the scales of the turbulent flow 343 increase downstream, the degree of interaction between bub344 bles and flow is expected to change along the jet. Far down345 stream the bubbles become effectively point-like and must 346 eventually behave as passive tracers because, since beyond 347 a certain distance from the injector, the smallest eddies will 348 become bigger than the bubbles. On the contrary, the situ349 ation is very different at the regions close to the jet inlet, 350 where bubbles are comparable to the jet diameter and to the 351 scale of velocity gradients. In those regions, bubbles will 352 necessarily be active in relation to the liquid flow field.

Measuring the Kolmogorov scales of turbulence (Lan4 dau and Lifshitz, 1987; Brennen, 2005) as well as the Stokes 5 bubble response time (Maxey et al, 1996; Brennen, 2005) 356 for the typical parameters of our experiments, we found that 35 bubbles become smaller than the larger scales of turbulence 358 at distances greater than $4 \mathrm{~cm}$, but they should not become 359 passive tracers until distances greater than $80 \mathrm{~cm}$. Being the 360 size of our experimental cell of $10 \mathrm{~cm}$, based on this sim361 ple scaling argument bubbles should in principle be active 362 and generate some appreciable back reaction to the flow for 363 most of the jet length. This effect should appear even bigger 364 taking into account that we can only measure velocities of 365 bubbles themselves, since we do not have any other tracer 366 on the flow. However, it is also important to realize that the 367 overall effect of the presence of bubbles on the statistics of 368 turbulence will depend also on the void fraction. For the typ369 ically small values of void fraction at hand, their effect may 370 still be quantitatively small. In fact, as already mentioned, 371 the results of Carrera et al (2008) showed that the mean flow 372 is not significantly affected by the presence of bubbles. Fur373 thermore, as we will see later, the statistical uncertainty of 374 our measurements does not allow us to detect significant de375 viations from the prediction of the numerical results under 376 the assumption of passive bubbles. We attribute this, in the 377 first place, to the small void fraction, which drops below the $37810 \%$ on gas after the first centimeter of jet (once we take 379 into account the initial opening of the jet due to the injector size), and also to the smallness of the effect of wakes created 1 behind bubbles at our small Reynolds numbers.

\section{Spatial structure of bubble jets}

Fig. 6 Contours of mean bubble concentration in an arbitrary scale, corresponding to a local diffusivity proportional to $k^{2} / \varepsilon$, as obtained by integration of the Fokker Planck equation by using the CFD results.

334 velocity decay roughly proportional to $\frac{1}{x}$, as well as its de- 3833.1 Computation of the spatial distribution of bubbles

335 pendence with the ratio $\frac{r}{x}$, which is maintained for the inter-

336 nal layers of the jet up to $\frac{r}{x} \simeq 0.10$. Outside these boundaries, 384 In order to try to resolve the possible bubble-turbulence cou-

337 the recirculation due to the finite size conditions become 385 pling in the jet, it is convenient to obtain, as a reference, re-

338 appreciable and, accordingly, the corresponding streamlines 386 sults for completely passive bubbles, i.e. when bubbles fol-

339 differ significantly.

387 low the local velocity field without modifying it. To this end 
388 we will use a stochastic model in which the bubble veloc389 ity is the result of the addition of the local mean flow plus a 390 stochastic diffusive contribution depending on the local in391 tensity of the turbulence.

392 To visualize the degree of inhomogeneity in the present 393 model regarding the diffusivity of bubbles, we plot in Fig. 5 394 the quantity $k^{2} / \varepsilon$, which is in principle proportional to the ef395 fective local diffusion coefficient of bubbles. The local dif396 fusivity is remarkably homogeneous in a certain central area 397 and abruptly drops on the sides, defining relatively clear-cut 398 jet boundaries. This drop in diffusivity is larger than one or399 der of magnitude in a relatively narrow layer. This explains 400 the small sensitivity of the results to small changes in $\sigma_{p}$ on 401 the determination of the diffusion coefficient $D_{P}$, since bub402 bles disperse through the whole central region, delimited by 403 this narrow boundary layer. For larger variations of $\sigma_{p}$ (of 404 around one order of magnitude) we reach the extreme be405 haviors possible for any scalar transport equation. In the case 406 with $\sigma_{p} \gtrsim 10$ the advection term predominates over diffu407 sion, impeding a significant dispersion of $P$ over the various 408 layers of the jet. In this case $P$ remains appreciable along the 409 central streamlines of the mean flow, thus strongly underes410 timating the opening angle of the bubble jet. On the contrary, 411 for $\sigma_{p} \lesssim 0.1$, diffusion predominates over advection, result${ }_{412} \mathrm{ing}$ in an overestimation of the opening angle and unrealistic 413 results near the injector, as a result of an extreme diffusivity. 414

In Fig. 6 we show the resulting bubble concentration 415 contours, obtained from the numerical integration of Eq. (14). ${ }_{416}$ As indicated earlier, bubble spreading is limited by the jet 417 boundaries, and the resulting spatial distributions are simi418 lar to those of experiments. Remarkably, this is not the case 419 if a homogeneous diffusivity is used (instead of one locally ${ }_{420}$ depending on $k^{2} / \varepsilon$ ). The use of a single value of diffusiv421 ity for the whole system results in a distribution of bubbles 422 that either opens in a very small angle (consistent with a ${ }_{423}$ scalar transport dominated by advection), or spreads out of ${ }_{424}$ the limits of the jet following an unrealistic behavior (corre425 sponding to a transport dominated by diffusion), depending 426 on the value taken for the diffusivity. An example of bubble 427 distribution $P$ in the case of constant diffusivity is shown in ${ }_{428}$ Fig. 7. We therefore conclude that, within the $k-\varepsilon$ model, an 429 inhomogeneous diffusivity is essential to capture the quali430 tative shape of the spatial distribution of bubbles.

\subsection{Bubble distribution. Experimental vs numerical results}

432 In order to compare the mean superficial density of bubbles ${ }_{433} \rho_{b}$ from the experimental snapshots with that from the nu434 merical results, we integrate the probability density of bub435 bles $P$ over the visual dimension $z$ in the form

$$
\rho_{b}(x, y)=C_{b} \int_{-\infty}^{\infty} P(x, y, z) d z .
$$

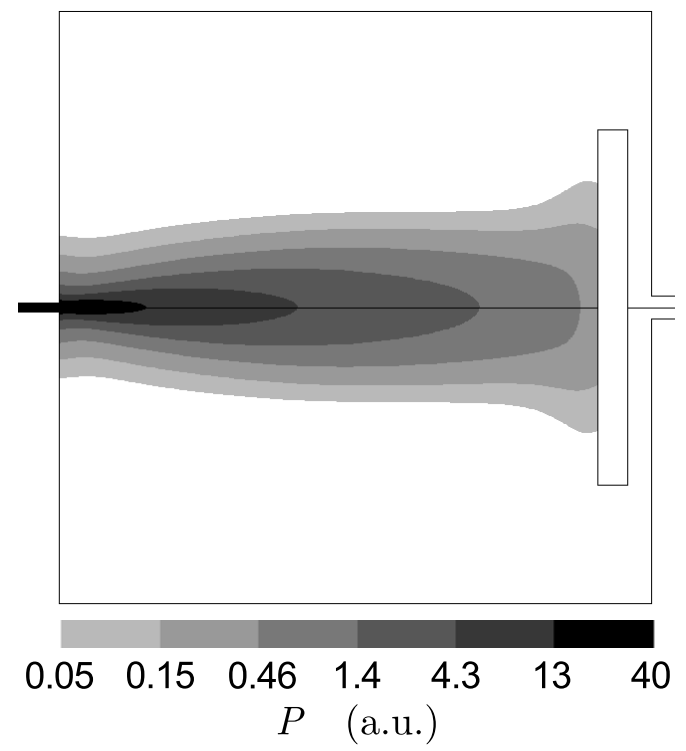

Fig. 7 Contours of mean bubble concentration in an arbitrary scale, corresponding to a homogeneous bubble diffusivity, as obtained by integration of the Fokker Planck equation. Unrealistic degree of diffusion is present close to the injector.

Since $P$ has been calculated in an arbitrary scale (we have ${ }_{37}$ not fixed the frequency of bubble injection), we introduce ${ }_{38} C_{b}$ as a constant to fix the density scale in the simulations ${ }_{39}$ in order to fit the experimental results. In Fig. 8 we com40 pare the experimental results with the numerical predictions ${ }_{41}$ of $\rho_{b}$ for different sections of the jet. The experimental val42 ues have been obtained by measuring the mean number of ${ }_{44}$ bubbles on small areas of the snapshots, averaged over the 44 whole duration of the microgravity conditions. The constant ${ }_{45} C_{b}$ in Eq. (17) has been fixed by imposing the same mean 446 number of bubbles on the section at $x=3 \mathrm{~cm}$ for both nu47 merical and experimental results. This number of bubbles is ${ }_{48}$ obtained by calculating the area below the curves in Fig. 8 49 at that distance.

As discussed in Carrera et al (2008), the experimental protocol to generate a uniform slug flow requires to start 42 injecting bubbles some time prior to the microgravity conditions. This is done in order to avoid the relative long tran${ }_{454}$ sients that precede a stationary generation of a uniform slug 455 flow. The downside of this procedure is that the gas injected 456 during normal gravity conditions is accelerated due to buoy457 ancy forces and drags some of the liquid, producing a weak, 458 residual liquid flow. Although buoyancy forces disappear 459 immediately at the start of the microgravity conditions, a slow relaxation of this residual flow remains, breaking the ${ }_{61}$ cylindrical symmetry of the jet and giving it a slight inclina462 tion upwards. This can be observed in the small lateral shift 463 of the experimental measures in Fig. 8. Also, the opening 464 angle of the bubble jet seems to be slightly smaller in the 465 simulations, as it can be observed in the figure at high dis- 

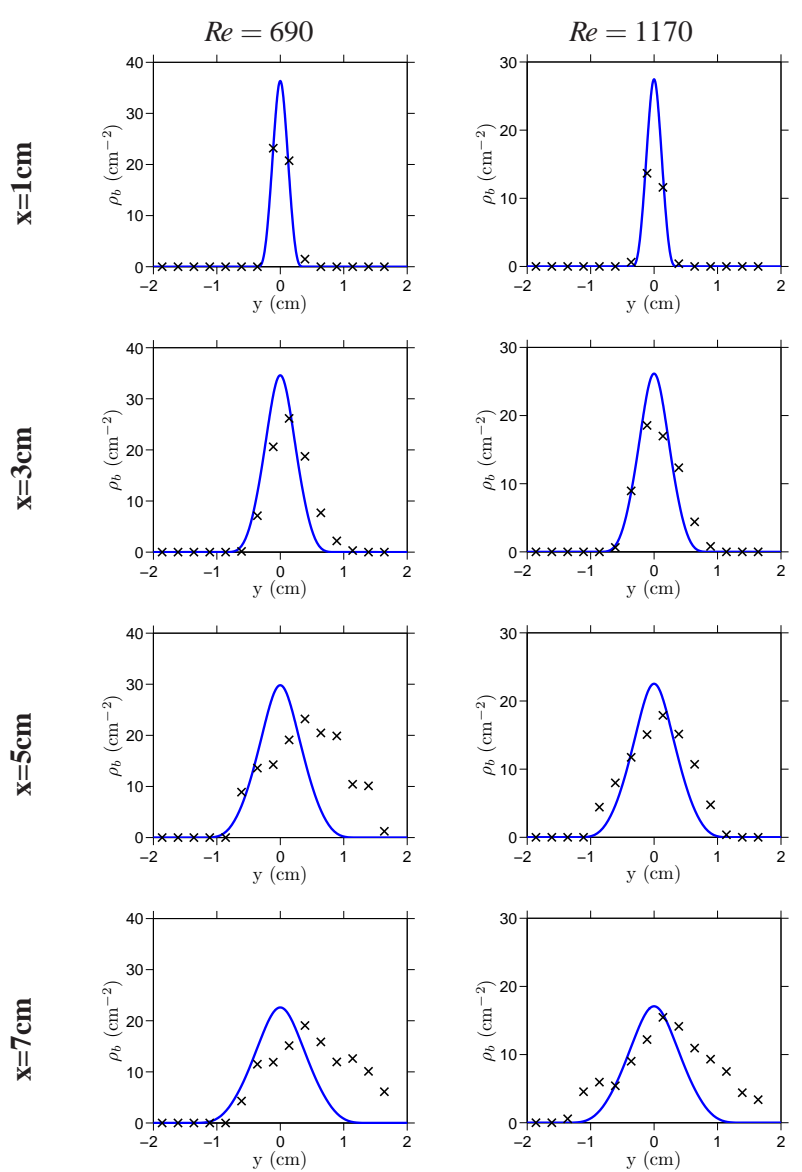

Fig. 8 Superficial density of bubbles at various sections of a jet ( $x={ }_{47}$ $1 \mathrm{~cm}, 3 \mathrm{~cm}, 5 \mathrm{~cm}$ and $7 \mathrm{~cm}$ ) for the cases of jets with $R e=690$ and $R e=$ 1170. Solid lines correspond to simulations and crosses to experimental results

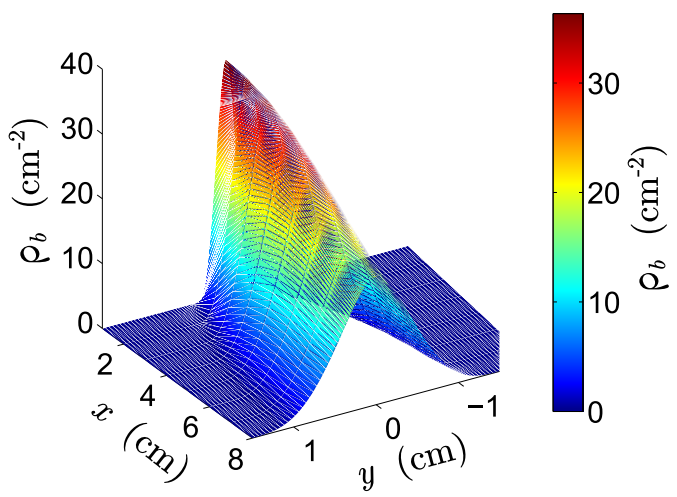

Fig. 9 Superficial density of bubbles $\rho_{b}\left(\mathrm{~cm}^{-2}\right)$ obtained from a simulation with $R e=690$, for all points on the projected $x y$ plane

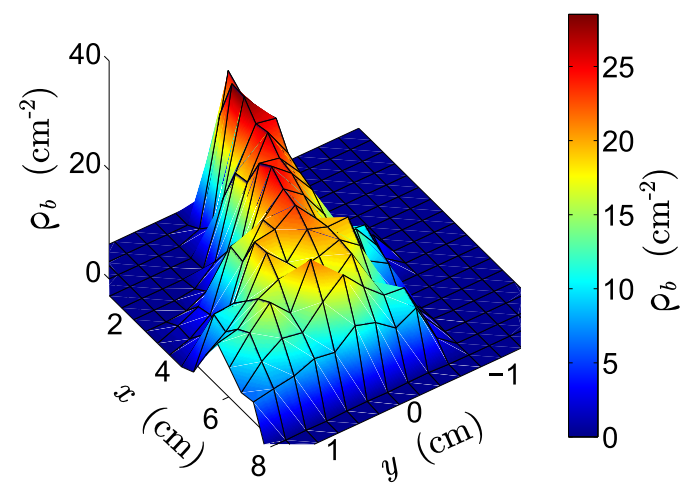

Fig. 10 Superficial density of bubbles $\rho_{b}\left(\mathrm{~cm}^{-2}\right)$ obtained experimentally in the case of $R e=690$, for all points on the projected $x y$ plane

466 tances from the injection point (i.e., $x=5 \mathrm{~cm}$ and $x=7 \mathrm{~cm}$ ), 467 arguably produced by the real effect of the finite size con468 ditions of our experimental cell. With the above disclaimers 469 and taking into account that the statistics of the data is nec470 essarily limited because of the restricted access to the mi471 crogravity conditions, the experimental data fit reasonably 472 well with the numerical prediction of our model, in particu473 lar in the intermediate range of distances to the inlet, when 474 the prediction of the model is most accurate. At the end of 475 the jet, the cumulative effect of the symmetry-breaking spu476 rious flow associated to the normal-gravity preparation of 477 the initial condition is most pronounced.

In Figs. 9 and 10 we show a 3D representation of the su79 perficial density of bubbles $\rho_{b}$, but in this case for any point 480 of the projected $x y$ plane, corresponding to all the points 481 where the data can be measured from the experimental snap482 shots.

\section{Velocity statistics and jet boundaries}

\section{${ }_{484} 4.1$ Mean velocity. Experimental vs numerical results}

${ }_{485}$ As before, since we cannot know the $z$ coordinate of the 486 bubbles position, we need to integrate the numerical pre${ }_{487}$ dictions over that dimension. In this case, one must take 488 into account that not all planes at different depths contribute 489 equally to the statistics. Indeed, layers where there are more 490 bubbles will contribute more significantly. Accordingly, in 491 order to compare the experimental velocity profiles $\left\langle u_{x}^{\exp }\right\rangle$ 492 with the numerical results obtained by numerical computa${ }_{493}$ tions $\left\langle u_{x}^{\text {sim }}\right\rangle$, it is necessary to introduce this projection ef494 fect into the simulation outcome. The way of achieving this 495 is by integrating the velocity of the flow $u_{x}(x, y, z)$ over the 496 visual dimension $z$ with the help of a weight factor $P^{\star}(x, y, z)$ 497 which stands for the proportion of bubbles at each point. $P^{\star}$ ${ }_{498}$ corresponds to the probability density of bubbles $P(x, y, z)$ 

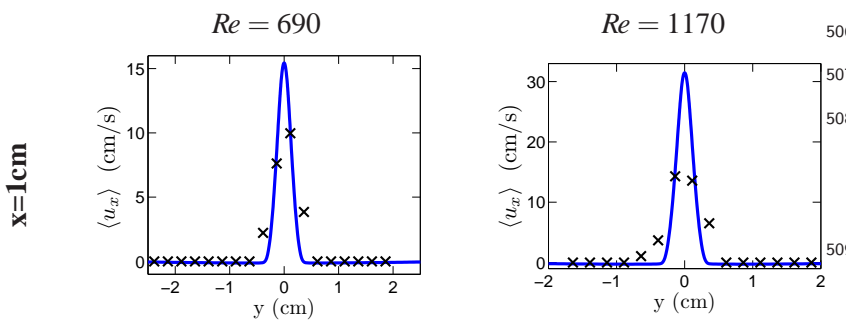

Now, we describe the velocity $u_{x}$ as the sum of a mean
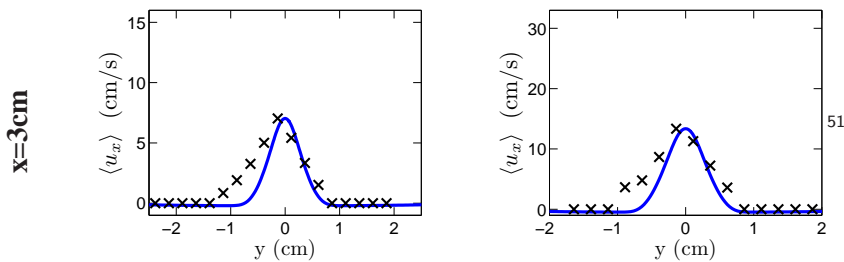

$\left\langle u_{x}(x, y, z, t)\right\rangle=U_{x}(x, y, z)+\left\langle u_{x}^{\prime}(x, y, z, t)\right\rangle=U_{x}(x, y, z)$

$u_{x}(x, y, z, t)=U_{x}(x, y, z)+u_{x}^{\prime}(x, y, z, t)$
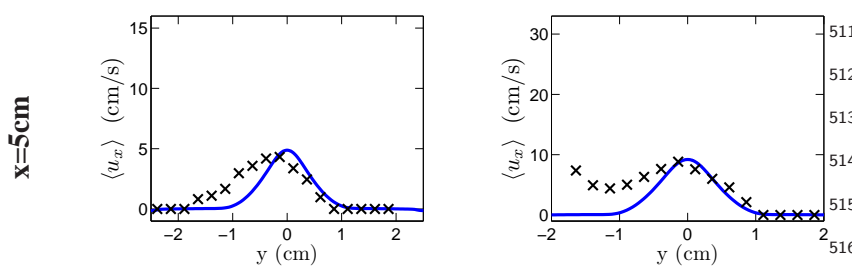

Applying it to Eq. (21) we finally obtain

$\left\langle u_{x}^{\operatorname{sim}}\right\rangle=\int_{-\infty}^{\infty} d z P^{\star}(x, y, z) U_{x}(x, y, z)$
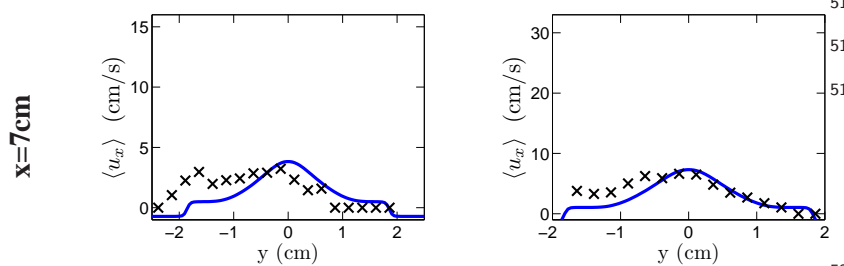

Due to the inherent uncertainty on the actual Reynolds number injected in the experiments, which may slightly fluctuate and deviate from the nominal value in a rather uncontrolled way, we have left an overall factor on the velocity scale of the simulations as an adjustable parameter. Since the structure of the jet should be equivalent for small injection vari17 ations, we scaled the velocity results of the simulations so that the maximum velocity $\left\langle u_{x}^{\text {sim }}\right\rangle$ in the section $x=3 \mathrm{~cm}$ 9 coincide with the experimental measurements, i.e.,

$$
\left\langle u_{x}^{\operatorname{sim}}(x=3 \mathrm{~cm})\right\rangle_{\mathrm{Max}}=\left\langle u_{x}^{\exp }(x=3 \mathrm{~cm})\right\rangle_{\mathrm{Max}} .
$$

Fig. 11 Mean axial velocity at various sections of a jet $(x=1 \mathrm{~cm}, 3 \mathrm{~cm}$,

520 For the case with $R e=690$ the simulated velocities have lines correspond to simulations $\left\langle u_{x}^{\text {sim }}\right\rangle$ and crosses to experimental re- 523 to all measured observables corresponding to the same exsults $\left\langle u_{x}^{\exp }\right\rangle$

${ }_{524}$ periment. In Fig. 11 we compare the numerical results with 525 the experimental data from our measurements.

499 normalized over the visual dimension $z$ in the form

$$
P^{\star}(x, y, z) \equiv \frac{P(x, y, z)}{\int_{-\infty}^{\infty} P(x, y, z) d z},
$$

5264.2 Velocity fluctuations. Experimental vs numerical results

500

$$
\int_{-\infty}^{\infty} P^{\star}(x, y, z) d z=1
$$

501 Then, the projected mean velocities of the flow, given by the 502 simulations are

$$
\left\langle u_{x}^{\operatorname{sim}}\right\rangle=\left\langle\int_{-\infty}^{\infty} d z P^{\star}(x, y, z) u_{x}(x, y, z, t)\right\rangle,
$$

(20) ${ }_{535}$ visual direction already contained in the mean flow, which

536 will already give a finite contribution even if the flow is lam503 which, under permutation of the order of the dimensional 504 integration and the statistical mean " \langle\rangle ”, yields

$$
\left\langle u_{x}^{\operatorname{sim}}\right\rangle=\int_{-\infty}^{\infty} d z P^{\star}(x, y, z)\left\langle u_{x}(x, y, z, t)\right\rangle
$$

$$
\left(\sigma_{x}^{\operatorname{sim}}\right)^{2} \equiv\left\langle\left(u_{x}^{\operatorname{sim}}\right)^{2}\right\rangle-\left\langle u_{x}^{\operatorname{sim}}\right\rangle^{2}
$$

538 and using the relations previously seen in Eqs. (20) and (21), 539 we find 

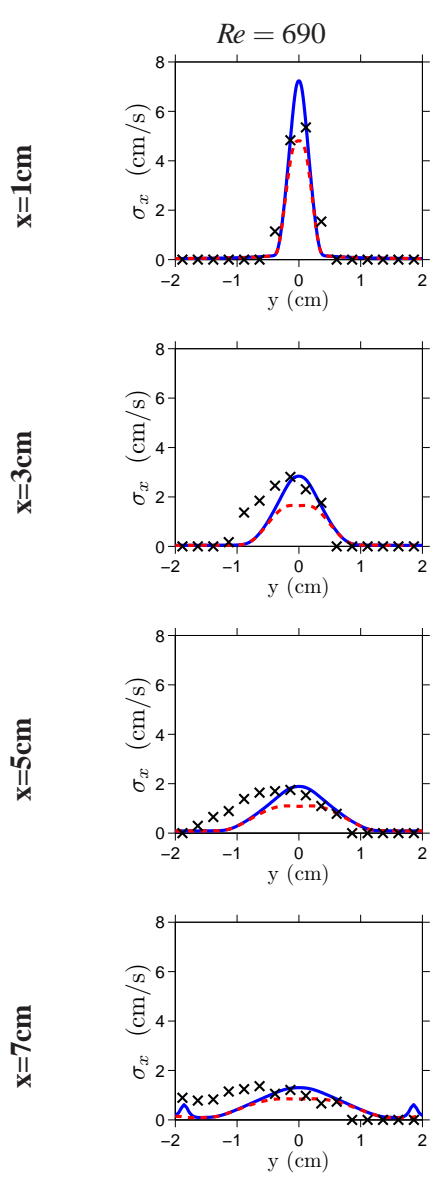

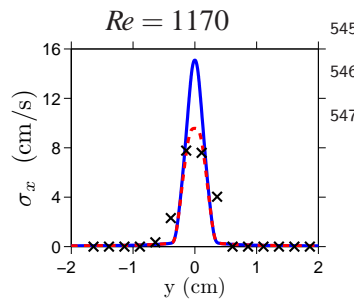

545 which, when introduced into Eq. (27) and after rearranging, 546 allow us to express the magnitude of the velocity fluctua${ }_{547}$ tions of bubbles $\sigma_{x}^{\text {sim }}$ as

$$
\left(\sigma_{x}^{\operatorname{sim}}\right)^{2}=\int_{-\infty}^{\infty} d z P^{\star} U_{x}^{2}-\left(\int_{-\infty}^{\infty} d z P^{\star} U_{x}\right)^{2}+\frac{2}{3} \int_{-\infty}^{\infty} d z P^{\star} k
$$

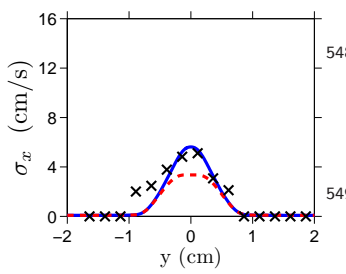

This equation can actually be expressed as

$$
\begin{aligned}
& \left(\sigma_{x}^{\operatorname{sim}}\right)^{2}=\sigma_{0}^{2}+\sigma_{k}^{2}, \\
& \sigma_{0}^{2}=\left(\int_{-\infty}^{\infty} d y P^{\star} U_{x}^{2}\right)-\left(\int_{-\infty}^{\infty} d y P^{\star} U_{x}\right)^{2},
\end{aligned}
$$

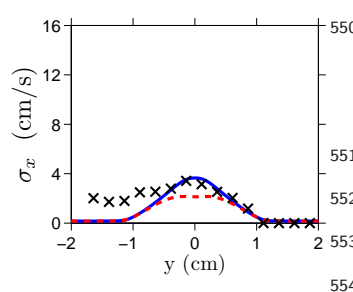

$\sigma_{k}^{2}=\frac{2}{3} \int_{-\infty}^{\infty} d z P^{\star} k$

551 In these expressions, $\sigma_{0}$ stands for the magnitude of the ap552 parent fluctuations due to the 3D structure of the jet, already 553 present for the a mean flow and which arise from the com554 parison of mean velocities at layers of different depth along

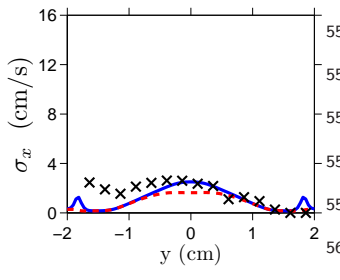
555 the visual line. On the other hand, $\sigma_{k}$ stands for the projec556 tion of the intrinsic fluctuations of the velocity at the differ557 ent layers of the jet, that is, those due to turbulence.

$558 \quad$ In Fig. 12 we compare the velocity fluctuations of the 559 experimental data with the numerical predictions calculated 500 with Eq. (31), with no additional fitting parameter, since the 561 velocity scale has already been fitted using the velocity mea-

Fig. 12 Velocity fluctuations at various sections of a jet $(x=1 \mathrm{~cm}, 3 \mathrm{~cm}, 562$ surements.

$5 \mathrm{~cm}$ and $7 \mathrm{~cm}$ ) for $R e=690$ and $R e=1170$. Solid lines correspond to simulations $\sigma_{x}^{\text {sim }}$ and crosses to experimental results $\sigma_{x}^{\exp }$. Dashed red lines correspond to $\sigma_{k}$, defined on Eq. (34)

Dashed lines show the value of $\sigma_{k}$ as defined in Eq. (34), 564 to illustrate the magnitude of the intrinsic velocity fluctua565 tions due to turbulence in relation to the apparent ones. As 566 for the measurements on the bubble spatial dispersion, for 567 both the measurements of mean values and dispersion of 568 bubbles velocities, the prediction of the $k$ - $\varepsilon$ model is also 569 reasonably accurate, within the inherent uncertainties of the 570 experimental data, and taking into account the symmetry $\left(\sigma_{x}^{\operatorname{sim}}\right)^{2}=\left\langle\int_{-\infty}^{\infty} d z P^{\star} u_{x}^{2}\right\rangle-\left\langle\int_{-\infty}^{\infty} d z P^{\star} u_{x}\right\rangle^{2}$ $=\int_{-\infty}^{\infty} d z P^{\star}\left\langle U_{x}^{2}+2 U_{x} u_{x}^{\prime}+u_{x}^{\prime 2}\right\rangle-\left(\int_{-\infty}^{\infty} d z P^{\star}\left\langle U_{x}+u_{x}^{\prime}\right\rangle\right)^{2}$ $=\int_{-\infty}^{\infty} d z P^{\star}\left(U_{x}^{2}+\left\langle u_{x}^{\prime 2}\right\rangle\right)-\left(\int_{-\infty}^{\infty} d z P^{\star} U_{x}\right)^{2}$. 572 residual flow from the preparation procedure under normal 573 gravity.

\section{(27) ${ }_{574} 4.3$ Jet boundaries}

540 541 bulence $k$ is

$k=\frac{1}{2}\left(\left\langle u_{x}^{\prime 2}\right\rangle+\left\langle u_{y}^{\prime 2}\right\rangle+\left\langle u_{z}^{\prime 2}\right\rangle\right)$,
575 Simulations seem to predict slightly smaller opening angles 576 of the bubble jet at large distances from the injection point. It (28) 577 is not clear if this could be attributed to an extra overspread578 ing of bubbles due to the stagnation disk or some other spu${ }_{542}$ and assuming for simplicity that turbulence is sufficiently 579 rious effect of the injection of bubbles in the stage prior to 543 isotropic, we obtain

$$
\begin{aligned}
& \left\langle u_{x}^{\prime 2}\right\rangle=\left\langle u_{y}^{\prime 2}\right\rangle=\left\langle u_{z}^{\prime 2}\right\rangle, \\
& k=\frac{3}{2}\left\langle u_{x}^{\prime 2}\right\rangle,
\end{aligned}
$$$$
544
$$

580 microgravity. In any case, one should take into account that 581 the boundary of the turbulent jet may not be well described ${ }_{582}$ within the frame of a $k$ - $\varepsilon$ model, because the latter implies ${ }_{583}$ a smooth variation of the properties $k$ and $\varepsilon$, whereas in re(30) 584 ality at the boundary between the jet and the laminar flow 


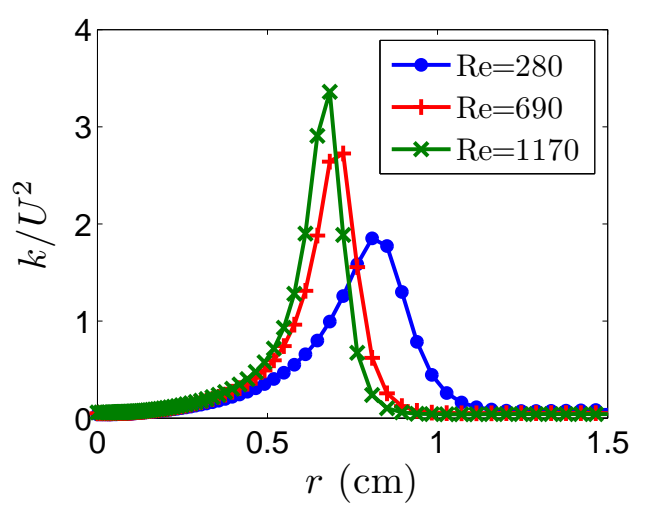

Fig. 13 Profiles of $k / U^{2}$ in function of the radial distance, at $\mathrm{x}=3 \mathrm{~cm}$

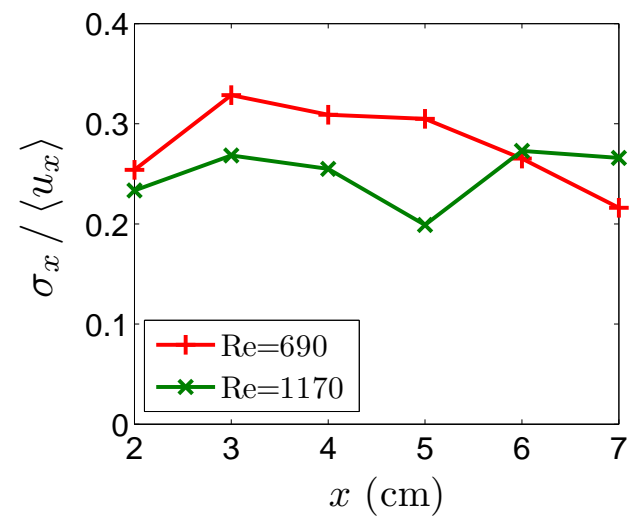

Fig. 14 Experimental measures of relative velocity fluctuations at the edges of the bubble jet

585 there can be significant changes of local flow characteris586 tics. Moreover the boundary between the turbulent jet and 587 the laminar flow is expected to fluctuate over time, and inter588 actions between bubbles and flow could be important at that 589 fluctuating boundary, for instance ejecting bubbles out of 590 the turbulent part of the jet. Consequently, the average effect 591 on the bubble dispersion and velocity statistics displayed by 52 bubbles near the jet boundary is likely to be missed by our simple model. In fact, when looking at the diffusion coef59 ficient $k^{2} / \varepsilon$ of the model, plotted in Fig. 5, we find a fast decay of this magnitude in a narrow distance, but this is still a smooth spatial variation and, most importantly, constant in time. It is thus not surprising to find deviations from the pre-

598 diction of the model in the experimental observation made ${ }_{64}$ 599 on the margins of the jet.

One way to define a the position of the jet boundary is 65 01 to assume that turbulence effectively vanishes past a certain ${ }_{65}$ 02 threshold value of $k$. In Fig. 13 we show the relative fluctua- ${ }_{65}$ tions of the flow for three simulations with different degrees 654 to the degree of approximation that is consistent with the in604 of turbulence. If we argue that the flow becomes laminar 655 herent uncertainty of the experiments, such interactions can 605 when the relative fluctuations of the flow $\left(k / U^{2}\right)$ drop be- ${ }_{656}$ be statistically neglected in the cases of the overall spatial 606 low a certain value, then we can see on the figure that the 657 distribution of bubbles, their mean velocity and the root607 tendency is that the radius of the jet (i.e., its opening an- 658 mean-square of their velocity fluctuations. This approxima-
${ }_{608} \mathrm{gle}$ ) increases for decreasing Reynolds number. We find the 609 same tendency if we define the boundary of the bubble jet 610 at some intrinsic property of the curve, for instance its in611 flection point. In this case, in addition, we also find that the ${ }_{612}$ relative fluctuations of the velocity at the jet margin as mea${ }_{613}$ sured by $k / U^{2}$ do increase with the Reynolds number.

${ }_{614}$ The above predictions of the model seem to be chal615 lenged by experimental observations. Indeed, Carrera et al 616 (2008) reported an opposite dependence of the measured 617 bubble opening angle (although measured very close to the 618 injector), which increased with $R e$ until saturation value for ${ }_{619} R e \approx 700$. In addition, the effect of Reynolds number on ${ }_{620}$ the relative velocity fluctuations at the jet boundary does ${ }_{621}$ not appear to be consistent with the model results. For this ${ }_{622}$ latter comparison, we have measured velocity fluctuations ${ }_{623}$ by carefully choosing bubbles one by one at the apparent 624 jet boundary, at maximal distance from the jet axis, in the ${ }_{625}$ projected plane of the images, thus minimizing the possi626 ble component along the visual coordinate $z$. In Fig. 14 we ${ }_{627}$ show the relative velocity fluctuations of the bubble veloc${ }_{628}$ ities measured on the bubbles at the margins of the exper629 imental images. The figure shows a weak decreasing ten${ }_{630}$ dency of the relative velocity fluctuations of bubbles at the ${ }_{631}$ boundaries when increasing Reynolds number, which would ${ }_{632}$ contradict the prediction from the $k-\varepsilon$ model defining the jet ${ }_{633}$ boundary at the inflection point. Although not fully conclu${ }_{634}$ sive, these observations seem to point out a limitation of the ${ }_{35}$ model to capture the behavior of bubbles near the jet bound6 aries, and consequently suggest active bubble-flow interac${ }_{637}$ tions in those regions. Much more involved CFD simulations should be invoked to be able to account more precisely 39 for the behavior at that level of detail, a problem that goes much beyond the scope of the present study.

\section{Conclusions}

A stochastic model that captures the essential statistics of bubble spatial dispersion in turbulent bubble jets formed by injection of capillary slug flows is presented. The treatment of bubbles as passive tracers with a local diffusivity associated to the $k-\varepsilon$ model seems to reasonably explain the ensemble dynamics of the bubbles. Numerical results obtained with our model compare well with experiments.

Simple scaling analysis comparing the bubble size and o the scales of turbulence indicate that the interaction between bubbles and its effect upon the carrying flow cannot be neglected in the regions relatively close to the inlet. However, our analysis shows that, even though potentially important to the degree of approximation that is consistent with the in- 
659 tion is expected to be progressively more accurate for in- 711 that of a turbulent multiply connected free-boundary prob${ }_{660}$ creasing distance downstream, since the flow is essentially 712 lem of great numerical difficulty.

661 self-similar with the scale fixed by the jet radius, while the

${ }_{662}$ bubbles become relatively smaller (effectively point-like) with ${ }_{713}$ Acknowledgements We gratefully thank Jordi Carrera for his key role

663 respect to the flow scales. 714 in initiating this line of research. We acknowledge the support from the

715 DLR, German Aerospace Center, for the funding of the drop tower ex-

Potential deviations of the effective model from the ex- ${ }_{716}$ periments that provided the raw data here analyzed. P.B., X.R. and J.C. 665 perimental statistics of bubble dispersion and velocity fluc- 717 acknowledge financial support from MICINN under projects FIS2010666 tuations have been detected at the margins of the jets. Indeed 718 21924-C02-02 and FIS2013-41144-P, and the Generalitat de Catalunya we found signs of a weak dependence of the relative velocity 719 under projects 2009-SGR-14 and 2014-SGR-878. L.R. acknowledges 22 financial support from Ministerio de Economia y Competividad (Spain) captured by the effective model. Similarly, the weak depen- 722 P.B. also acknowledges MCyT for a pre-doctoral fellowship.

dence of the jet opening angle predicted by the model dif-

71 fers from measurements from Carrera et al (2008). Although

72 the limited statistics of the experiments is not fully conclu- ${ }_{723}$ References

673 sive, these observations suggest that the boundaries of the

674 jet are regions where the bubble dynamics is most sensitive, ${ }_{724}$ Arias S, Ruiz X, Casademunt J, Ramírez-Piscina L, 675 and where inaccuracies of the model may be more apparent, 725 676 even relatively far downstream. This points to the possible 726 677 failure of the hypothesis of passive bubbles, assumed in the 727 model, and hence to the relevance of the bubble-flow inter- 728 79 actions at the boundaries of the jet.

González-Cinca R (2009) Experimental study of a microchannel bubble injector for microgravity applications. Microgravity Sci Technol 21(1):107-111

Arias S, González-Cinca R, Ruiz X, Ramírez-Piscina L, Casademunt J (2010) Characterization of the performance of a minibubble generator in conditions relevant to microgravity. Colloids and Surfaces A: Physicochemical and Engineering Aspects 365:52-55

${ }_{681}$ the ensemble statistics of many realizations of bubble jets, ${ }_{682}$ but cannot provide relevant information contained in the prop${ }_{683}$ erties of the actual bubble trajectories, for instance to define ${ }_{684}$ the probability of bubble encounters, and consequently of ${ }_{735}^{734}$ 685 potential coalescence events.

Bitlloch P (2012) Turbulent bubble suspensions and crystal growth in microgravity. Drop tower experiments and numerical simulations. PhD Thesis, Universitat de Barcelona

A more accurate description of the system should also ${ }_{737}$ Brennen CE (2005) Fundamentals of multhiphase flow, ${ }_{687}$ aim at a more realistic modeling of the bubble trajectories. 738 68 Diffusive trajectories are indeed too erratic on small scales 739 and overestimate significantly the probability of bubble en- 740 690 counters. Introducing a more realistic tracking of the flow 741 691 trajectories, even if still as passive tracers, should take into 742 ${ }_{692}$ account statistical correlations of the flow which would clearly $y_{43}$ ${ }_{693}$ modify the statistics of bubble encounters. This point has 744 ${ }_{694}$ remarkable practical relevance because reducing the degree ${ }_{745}$ 695 of bubble coalescence is important to keep the monodis- 746 ${ }_{696}$ persivity of the suspension, and ultimately the control of 747 697 the surface-to-volume ratio. Our jets do exhibit a remark- 748 ${ }_{698}$ ably low degree of bubble coalescence, a point that was al- 749 699 ready discussed by Carrera et al (2008). A full description 750 700 of the dynamics of suspensions of spherical bubbles, includ- 751 701 ing bubble-bubble interactions and bubble-flow interactions 752 702 could be approached with large scale Lattice-Boltzmann sim- 753 703 ulations, in the spirit of the work of Yin et al (2006). In the 754 704 case of bubble jets, however, the non-homogeneous condi- 755 705 tions along the jet makes this analysis very demanding. It 756 706 is particularly difficult to incorporate correctly the physics 757 707 of the two-phase flow right at the exit of the injector, where 758 708 bubbles may significantly deform due to the strong slowing- 759 709 down as they enter the cavity, and the variations of the flow 760 710 field are strong at the scale of bubbles. There the problem is 761 Cambridge University Press, chap 1, pp 19-51

Carrera J, Ruiz X, Ramírez-Piscina L, Casademunt J, Dreyer M (2008) Generation of a monodisperse microbubble jet in microgravity. AIAA Journal 46(8):2010 - 2019

Landau LD, Lifshitz E (1987) Fluid Mechanics, Pergamon Press, chap III, pp 102-140. Course of Theoretical Physics - Volume 6

Maxey M, Chang E, Wang L (1996) Interactions of particles and microbubbles with turbulence. Experimental Thermal and Fluid Science 12:417-425

National Research Council of the National Academies (2012) Nasa space technology roadmaps and priorities: Restoring nasa's technological edge and paving the way for a new era in space. http://www.nap.edu/ openbook.php?record_id=13354

Schlichting H (1979) Boundary-Layer Theory, McGrawHill Classic Textbook Reissue. Seventh Edition, chap XXIV, pp 729-757

Shih TH, Liou WW, Shabbir A, Yang Z, Zhu J (1995) A new $k-\varepsilon$ eddy viscosity model for high reynolds number turbulent flows. Computers \& Fluids 24(3):227 - 238

Versteeg H, Malalasekera W (1995) An introduction to computational fluid dynamics. The finite volume method, Pearson Prentice Hall, chap 3, pp 41-84 
${ }_{762}$ Yin X, Koch DL, Verberg R (2006) Lattice-boltzmann 763 method for simulating spherical bubbles with no tangen-

764 tial stress boundary conditions. Phys Rev E 73:026,301-1

765 to $026,301-13$ 